\title{
EDITORIAL
}

\section{Intellectual disability: an unresolved world health challenge}

Tntellectual disability (ID) is a frequently and highly 1 disabling health condition. Its prevalence is between 0.5 and $3 \%$ of the general population; it presents both an elevated physical as well as psychological comorbility and implies consequences for the person and their family throughout the life cycle. It is, thus, not surprising that ID represents the principal cause of health and social expenses in developed countries such as the United Kingdom and Holland. This fact should lead to the development of specific healthcare programs for this group, but, paradoxically, ID is the most forgotten of public health programs. ${ }^{1}$ This situation is particularly serious in countries with small and medium economies where the ID rate may be four times that found in countries with advanced economies. The etiology of ID is preventable in many cases, since it is related to the consumption of neurotoxic agents such as lead, nutritional deficits, or problems during gestation, childbirth or premature development. The impact of health policies aimed at correcting the causes of ID could be enormous. It has been estimated that ID and cardiovascular diseases derived from lead exposure may be attributable to $1 \%$ of the global illness burden. ${ }^{2}$ Furthermore, there is a large gap in healthcare for this group of disorders, including in first-world countries. In Spain, the etiology of ID is unknown in $60 \%$ of the cases, in spite of available means for adequate genetic screening and a high degree of general pediatric care. Health needs are especially severe in the adult and elderly population with ID, which in many countries, when not in a state of semi-reclusion at home or in a residence, continue to receive care in large psychiatric institutions without adequate care. This lack of care is also reflected in the lack of education in health profes- sions with respect to ID. The time dedicated to training medical students about ID is less than one elective hour in the majority of countries, and most educational programs in psychiatry do not include ID, in spite of the fact that, as indicated by The WHO atlas on intellectual disability (Lecomte and Mercier, in this issue), roughly one-third of the persons with ID present serious psychiatric problems and that, worldwide, the psychiatrist is the first health professional to provide care to persons with ID. In light of the data and the current capacity for intervention, it is not acceptable that ID continues to be the most unresolved issue for national and international health systems.

Fortunately, a change in this tendency has been produced in recent years. Diverse international associations such as the IASSID, NADD and MHDI have taken on an important role in the visualization of ID as a worldwide health and social problem and they have aided in the dissemination and use of international evaluation and diagnostic instruments such as the recent Psychiatric Diagnostic Manual on Intellectual Disability. ${ }^{3}$ Some international research networks, such as IDRESNET, POMONA and WHO, have facilitated the development of health indicators and international comparison systems for services. This issue of the journal Salud Pública de México covers both general aspects and their relations with health habits from a broad perspective, as well as the provision of services, health policy and ethical factors. For the first time, a public health journal is dedicating an issue to ID, which constitutes a milestone for this metasyndrome to be considered a public health priority.

Luis Salvador-Carulla*

\footnotetext{
* Professor of Psychiatry, University of Cadiz (Spain). President, Intellectual Disability Section, World Psychiatric Association.
} 


\section{References}

I. Salvador-Carulla L, Bertelli M. Mental Retardation or Intellectual Disability:Time for a Conceptual Change? Psychopathology 2008;4I: 10-16.

2. Fewtrell LJ, Pruss-Ustun A, Landrigan P,Ayuso-Mateos JL. Estimating the global burden of disease of mild mental retardation and cardiovascular diseases from environmental lead exposure. Environ Res 2004;94(2): 120-33.

3. Fletcher RJ, Loschen E, Stavrakaki C, First M. Diagnostic Manual. Intellectual Disability:A Textbook of Diagnosis of Mental Disorders in Persons with Intellectual Disability. National Association for the Dually Diagnosed (NADD) in association with the American Psychiatric Association (APA), Boston, 2007. 